

\title{
A Game Design Method for Therapeutic Games
}

Stéphanie Mader, Guillaume Levieux, Stéphane Natkin

\section{To cite this version:}

Stéphanie Mader, Guillaume Levieux, Stéphane Natkin. A Game Design Method for Therapeutic Games. 2016 8th International Conference on Games and Virtual Worlds for Serious Applications (VS-Games), Sep 2016, Barcelona, Spain. pp.1-8, 10.1109/VS-GAMES.2016.7590333 . hal-02456092

\section{HAL Id: hal-02456092 https://hal-cnam.archives-ouvertes.fr/hal-02456092}

Submitted on 27 Jan 2020

HAL is a multi-disciplinary open access archive for the deposit and dissemination of scientific research documents, whether they are published or not. The documents may come from teaching and research institutions in France or abroad, or from public or private research centers.
L'archive ouverte pluridisciplinaire HAL, est destinée au dépôt et à la diffusion de documents scientifiques de niveau recherche, publiés ou non, émanant des établissements d'enseignement et de recherche français ou étrangers, des laboratoires publics ou privés. 


\title{
A Game Design Method for Therapeutic Games
}

\author{
Stéphanie Mader, Guillaume Levieux and Stéphane Natkin \\ Laboratoire CEDRIC \\ Conservatoire National des Arts et Métiers \\ Paris, France \\ http://cedric.cnam.fr \\ stephanie.mader@cnam.fr, guillaume.levieux@cnam.fr, stephane.natkin@cnam.fr
}

\begin{abstract}
One of the most problematic issues in healthcare is the patient's lack of adherence to the therapy. Patients' motivation is indeed hard to maintain when they have to execute repetitive, boring or tedious exercises. In such cases, they tend to practice less regularly and even to entirely give up the therapeutic protocol.

Fortunately, therapeutic exercises can very often be turned into compelling games. Such therapeutic games are considered as a very promising solution to the patient adherence problem.

Yet, therapeutic games are very complex to design : 1 . the gameplay is particularly constrained, e.g. the game has to both motivate the patient and provide the therapeutic effects 2 . the game must be evaluated on both its medical and motivational results, and 3. relevant health knowledge is hard to share between health experts and game designers.

In this paper, we propose a game design method for therapeutic games that provides guidance for every step of the design, along with tools for every design challenges we identified.
\end{abstract}

\section{INTRODUCTION}

Games are known for being compelling to the extent of motivating their players for many hours [1]. The principle of combining game and therapy arises from the necessity to motivate patients to follow their therapeutic protocol.

Indeed, according to the World Health Organisation report on adherence, regularity and protocol compliance are strongly correlated with a therapeutic protocol efficacy [2]. Unfortunately, the same report estimates that adherence only averages $50 \%$ regarding long-term therapy for chronic illnesses in developed countries. The report explains that: "motivation, which drives sustainable good adherence, is one of the most difficult elements for the health care system to provide in the long term"[2].

This adherence issue is not limited to life-style changes, medications and chronic diseases. According to Bassett, approximately $65 \%$ patients are not regular or non adherent at all to physiotherapy rehabilitation [3]. Therapeutic activities are designed to be effective, and patients often have to do boring or tedious exercises, which may diminish their motivation and lead them to practice less regularly or even stop the protocol [4], [5].

As it is possible to turn existing exercises into games, video games are currently explored as a way to maximize the patients' motivation. Examples of therapeutic games are already numerous and the amount of research on these specific games as well as on the positive effects of play on health is growing. For instance, Anguera et al. proved that their game was able to reduce the multi-tasking cost in older adults [6]. The trained older adults (60-85 y.o.) attained performance levels beyond those of untrained 20 year olds. Others studies report the use of video games to alleviate pain in burned patients [7] or to improve motor control after a stroke [5].

But even if therapeutic games seem to be very promising, they are still very complex to design and develop. The game designer has to create a motivating and efficient game but often lacks the medical knowledge necessary for this design. This is particularly important for the gameplay (i.e. the player's objectives and the actions s/he can do to attain the objective [8]). The gameplay is the very heart of a video game, and in therapeutic games, it must provide the therapy through relevant game actions while also being able to sustain the patients motivation. From now on, we will qualify such gameplay as a therapeutic gameplay.

In this paper, we propose a game design method for therapeutic games. This method is addressed to game designers and proposes a design process with tools to address the main design challenges of therapeutic game design.

First, we introduce the notions of therapeutic games, gameplay, and game design. Then, we present existing game design methods for entertainment and serious games. After that, we provide an overview of the method and its components. Then, we explain the main design challenges of therapeutic game design. In section VIII, we present our method and tools. Finally, we discuss the limits of our method and describe our future works.

\section{Therapeutic GAMES \& GAMEPLAY}

\section{A. Therapeutic games}

According to the McGraw-Hill Concise Dictionary of Modern Medicine, therapy is "a general term for any form of management of a particular condition ; treatment intended and expected to alleviate disease or disorder; any technique of recovery, which may be medical, psychiatric, or psychological'[9].

As a consequence, we define therapeutic games as follow: games that produce a direct, expected, and intended therapeutic effect on patients playing them. This therapeutic effect may be to alleviate, to improve or to heal the specific condition of the patients.

With this definition, we emphasize two important aspects of therapeutic games. 
First, the therapeutic effect is intended and expected, which means that medical proofs must confirm that this patient will benefits from this game. It is a way to state firmly that it is fundamental for therapeutic games to be thoroughly validated.

Second, the therapeutic game has to produce a direct therapeutic effect. This rule is important as we identified that most Games For Health may try to indirectly improve the health of someone, but not necessarily the player. In these games, the health effects derives from the behavior change (e.g. quit smoking, taking his/her medication), not directly from the play session. Some health games are not even intended to be played by the patient him/herself but target his surroundings, e.g. showing them how to behave with a depressive friend.

In this paper, we focus on therapeutic games, which are, by our definition, the only ones to improve directly the health of their players.

\section{B. Gameplay \& Therapeutic Gameplay}

Gameplay is at the very center of a game, and can be considered as the designed relationship between the player and the game. Gameplay defines how the player and the game interact with each other, it defines both the structure and purpose of the player interaction with the game. Most of the time, this purpose is stated in terms of player's aesthetic experience [10]. To create this experience for the player, an important part of gameplay design is to define the player's objectives in the game and the means at his/her disposal to attain those objectives [8]. As a result, a thorough description of a gameplay should define every action the player does to attain the objectives. Those actions may be, for instance, to look at the screen, to identify an enemy, to aim or to press buttons.

Our previous definition of therapeutic games implies that the player improves his/her health directly by interacting with the game, and thus that a therapeutic game provides a therapeutic gameplay. We define a therapeutic gameplay as a gameplay designed to lead the player to interact with the game through at least one action that is beneficial for his/her health. For example, to complete the game objective (e.g. collecting ten apples), the player has to execute a specific movement with his/her arms (e.g. extending his/her arms). If this movement improves his/her health conditions (e.g. improving the arm's flexibility), the therapeutic effect is due to the player interacting with the game.

According to Salen and Zimmerman, "game design is the process by which a game designer creates a game, to be encountered by a player, from which meaningful play emerges" [11]. For a therapeutic game, the game designer needs to define a gameplay from which meaningful, motivating play sessions will emerge, but that will also provide a very specific therapeutic effect. To reach such a complex design goal, the entire design and production process needs adaptations to take into account both objectives. In the next section, we examine the production process of video games, along with important aspects of game design, and existing game design methods.

\section{RELATED WORKS}

Game design is one aspect of the overall production process of a video game. In video games, the production process is usually composed of five phases: concept, pre-production, production, quality assurance and maintenance [12]. The game design objectives progress according to its own process, but also adapt to the production stage. For instance, as the production advances, game design changes should be of less and less importance [12].

Most authors agree that game design is a user-centric iterative process composed of three steps: design, prototype, and evaluate [11], [13], [12], [14]. Beyond that, few authors propose specific design process (e.g. Fullerton's prototyping process). As stated by Schell, there is no definite model of game design, game designers compose their own set of principles and rules [15]. Game designers and researchers add to the game design knowledge by providing design recommendations or patterns based on their experiences (e.g. [16]), player models (e.g. [17], [18]), or game models (e.g. [19]).

As game design is a user-centric process, player models are very important as they help to understand the player for which the game is designed. Player models include for instance play preferences and motivation.

Game models are about describing and analyzing games, they may serve to classify games (e.g. game genres), to analyse game components (e.g. Malone's taxonomy of intrinsically motivating game features), or to explore a specific features (e.g. gameplay).

As a consequence, game designers usually compose their own toolbox of methods and models and create their own tools on top of existing ones.

The situation is similar for serious games: authors proposed methods, models, and recommendations (e.g. use of adaptive difficulty algorithms [5]). To our knowledge, there is only one game design method that is specific to therapeutic games: Benveniste's Incremental Design.

Incremental Design is an adaptation of Action Research for therapeutic games [20]. It is a four phases process. The first step is to develop an a priori robust and simple prototype based on discussions with experts. Then, the second and third phases are iterative: perform small-scale evaluations of the prototype with patients during a sufficient amount of time and then adjust the prototype by small changes based on the feedbacks. For this, Benveniste emphasizes the importance of testing any modifications even if they seem harmless. Finally, the last phase is to freeze the design, refactor the source code and start dissemination. Benveniste explains that therapeutic evaluation should not start before the fun is guaranteed.

Incremental Design is very relevant for therapeutic games. Yet, this method is only applicable when the game designer has a constant access to patients which is not always the case. Plus, we think that a more extensive process is needed to efficiently guide the work of game designers. Indeed, difficulties such as exchanging information with health experts or designing the gameplay should be guided with specific tools. 
In other serious game fields, such as educational games, methods have been proposed. These methods mostly define the role and responsibilities of the game designer and the domainspecific experts, along with the main production steps (e.g. [21], [22]). Some of these methods also include data-model about the player or the game to facilitate the design or the exchange of information. But, as those models are domainspecific, they are not directly applicable to therapeutic game.

\section{OVERVIEW OF THE DESIGN METHOD FOR THERAPEUTIC GAMES}

In this paper, we present a design method for therapeutic games, along with it foundations (i.e. which design challenges we intend to solve). This section aims at giving a general overview of the method and its components.

The design method contains a sequential process of three phases. The phase 2 and 3 contain three iterative steps.

- Phase 1: Investigating the problem with health experts

- Phase 2: Designing the gameplay

- Step 2.1. Gameplay design and formalisation

- Step 2.2. Gameplay prototyping

- Step 2.3. Playtest

- Phase 3: Prototyping the therapeutic game

- Step 3.1. Game design

- Step 3.2. Game prototyping

- Step 3.3. Medical validation \& playtests

We constructed different tools to help the achievement of each phases and steps. The player/game/therapy model ( $\mathrm{P} / \mathrm{G} / \mathrm{T}$ model) is used during the first phase to help the exchange of information between health experst and game designers. The P/G/T model contains a therapy model, a game model, and a player-patient model, particularly a sub-model is dedicated to the patient's abilities to play. The gameplay action model is used during the step 2.2 in order to describe the gameplay in details. In step 2.3., the player in-game skills evaluation tool serves to assess what patients are able to do in games and particularly to validate the gameplay designed during the previous step.

The details of these tools can not be included in this paper due to their length (e.g. patient's ability to play model is 17 pages long). They can be consulted in [23].

In the next sections, we use our P/G/T model to identify the challenges of therapeutic game design, then present them in details. After that, we present the components of the method and finally explain the design process.

\section{UNDERSTANDING THERAPEUTIC GAMES: THE P/G/T MODEL}

To better understand therapeutic games, we propose the player / game / therapy model (P/G/T model, an early version has been previously published in [24]). Indeed, a therapeutic game is a complex dynamic process, where the player, the game and the therapy interact with each other. The P/G/T model separates the three main aspects of therapeutic game into sub-models. Each sub-models contains a list of important elements to describe the related aspect.
First, the player-patient model serves to describe the person as a player and as a patient. The basis of the model is a list of general information: gender, age, entertainment tastes and play preferences. Then, the model contains specific elements about the patient's situation (e.g. where s/he lives, what help does s/he need on a daily basis) and a sub-model about the patient abilities to play (e.g. how much time can the patient stay sited in front of a computer, at which speed can $\mathrm{s}$ /he proceed information written on screen). Those data are necessary to design a game that is playable and enjoyable for the patient.

The game model serves to define the basis of the game: genre, platform and devices, short description of the story, the gameplay and the game world. It is a summary of the main aspects of the game that may be analysed in relation to the other aspects (i.e. player and therapy). The model is not the entire design of the game and should not replace the game design document.

Finally, the therapy model serves to describe the therapy that should be embedded into the game. Most of its content will be given by health experts and thereafter used as design constraints. The therapy model contains elements about the therapeutic objective (e.g. short and long-term effects), the therapeutic protocol (e.g. occurrence, frequency, duration), the context (e.g. place of the therapist), and efficiency (e.g. evidences that this therapy is efficient).

As an analysis tool, the main idea behind the P/G/T model is to 1 . analyse each aspects of a therapeutic game independently and 2. analyse the relations between each of them (i.e. playergame, player-therapy, and therapy-game).

This analysis can be done on existing therapeutic games to better understand their design and how the relations works. When performed during design, the $\mathrm{P} / \mathrm{G} / \mathrm{T}$ model serves as a reminder of important data to get (e.g. health information about the patient) or decide (e.g. which kind/genre of game will be made). Then, the collected data and the relation analysis serves to define the design constraints and allow to highlight design inconsistency (i.e. incompatibility between aspects).

The player-therapy relation defines if this therapy can improve the specific health condition of this patient. Game designers being seldom knowledgeable about those aspects, the health experts are responsible to validate the relevancy of the patient-therapy relation. The game designers are responsible of the two other relations: game-therapy and player-game.

The game-therapy relation defines if the game provides the therapy. If the game is not what provide the therapy, then it is not a therapeutic game. It may be a therapy making use of games as mediation tools or as rewards. As such our models and methods may not be relevant to them. For the game being what provides the therapy, it must have a therapeutic gameplay, which means that at least one game action provides the therapeutic effect on the patient. Designing the therapeutic gameplay is the most difficult and constrained design task of a therapeutic game. It requires strong knowledge in game design and health. For this particular aspect, game designers 
and health experts have to strongly collaborate.

Moreover, this relation can only be validated through medical experimentations. As the cost in time and money of such experimentation is high, they will not be done regularly, which means that an iteration on this aspect will be long, as opposed to the usual short cycle of video game design.

The player-game relation is about the compatibility of the game with the tastes and the abilities of the targeted players. This relation analysis determine 1 . if the game is motivating for the player and 2. if the player is able to play this game. Both those aspects are challenging in therapeutic games as they require the use of player's model that are not always preexistent. For instance, we have no model of player suffering from the Alzheimer's disease.

From a design perspective, the $\mathrm{P} / \mathrm{G} / \mathrm{T}$ model serves to collect the necessary information and analyze them to define the design constraints. Then, the game designer can find the sweet spot between what the player will enjoy while playing, what the player can do in games, and what is useful in games to provide the therapy (see fig 1).



Figure 1. Sweet spot of the design space in the P/G/T model

\section{Therapeutic Game Design Challenges}

While presenting the model, we used it to illustrate therapeutic game design challenges. The interdisciplinary nature of therapeutic games and the specificities of working with patients are the main reasons behind the 4 main design challenges we found:

- Designing a motivating game

- Exchanging information

- Designing a therapeutic gameplay

- Defining the process including the validation

\section{A. Designing a motivating game}

As stated by Ghozland, the player's motivation is what define how long s/he will play and if s/he will finish the game [25]. Player's motivation may be about different inherent appealing features of games, such as the challenges, the immersion into a game world and the social interactions [19], [18], [26]. As a consequence, for each game, game designers define their target player through a model to guide their design. Existing player's model are often about defining player's type according to their play preference. For instance, some players are more driven by the story content, while others like facing difficult challenges.

Two design challenges emerge from the objective of maximizing the patient's motivation to pursue his/her therapeutic protocol.

First, therapeutic activities are seldom interesting in themselves, they may be repetitive, boring, and tedious. The game designer has to turn them into games without losing their therapeutic effects and make them more motivating by applying design techniques. This is particularly linked to the design of the therapeutic gameplay.

Second, the target player is not necessary inside known boundaries. For instance, there is little data about what seniors enjoy while playing [27]. This issue complicates the selection of motivating features as game designers have to take supplementary steps to study what the patients like or dislike while playing.

As it is an important objective of therapeutic games, the patient's enjoyment and motivation while playing must be well-crafted and evaluated through playtests. If the game does not achieve its objectives of maximizing motivation it should be improved until it achieves them. This is why our process include steps like playtests to validate that the game is enjoyable and motivating for the patient.

\section{B. Exchanging information}

As stated before, the game designer must know about the therapy and the patient, particularly his/her abilities.

Game designers are seldom knowledgeable about health, they may not even know which information they need. For instance, if the game targets patients suffering from the Alzheimer's disease (AD), game designers may not intuitively ask information about their motor and perceptual functioning. This lack of data may later lead them to design a game not accessible to them, especially since AD population is mainly seniors and that ageing have effects on motor and perceptual functions.

Indeed, what usual players are able to do in game (e.g. at each speed they can identify a target and shoot at it) is difficult to define exactly, but designers rely on approximation based on their intuition and experience. Afterwards, they can calibrate the game with actual players. In therapeutic game, it is not only a question of tuning, but also of designing a gameplay that the patient may be able to do. For instance, the patient may not be able to identify a target on screen or to use a mouse to shoot at it. Thus, game designers of therapeutic games need a model of the patient's abilities.

On the other side, health experts are seldom game expert and while they have a lot of knowledge, they do not necessarily know which of these information are important to design a game. As it would be too time-consuming for the health 
experts to explain everything they know about the patient, they will select which information they give. By doing so, important information tend to be given only when the health expert analyses the design or the prototype and find something that will not work well according to his/her knowledge and insights. It would be preferable to avoid as much as possible this situation by having the information first and use it to constraint the design.

A way to help the timely transfer of knowledge is to provide the game designer with models containing lists of elements s/he should know. S/he can then ask health experts about the information s/he lacks. Our P/G/T model contains basic information about the therapy and the patient. However, if the therapy can be described through few chosen elements, the patient is more complex and a thorough description of patient's abilities to play is needed. In section VII, we present a model of the patient's abilities to play and a tool to evaluate more precisely what patients can do in games.

\section{Designing a therapeutic gameplay}

The gameplay is the most constrained aspect of a therapeutic game, it should by enjoyable and motivating and it should entice the player to do the game action affecting his/her health. Moreover, every game actions must be doable by the patient regarding his/her abilities.

This design challenge is tied to the exchange of information about the therapy and the patients, particularly the analysis of the patient's abilities. Theses data are used as design constrained while creating the gameplay. Then, the health expert should validate the gameplay regarding the therapeutic effects and assess if every actions are doable for the patient.

It is really difficult to convey a gameplay to non-specialists and this is even more complicated by the lack of methods to describe gameplay in details [23]. Thus, in section VII, we propose a way to decompose a gameplay into game actions in order to help the game designer to do the initial design, but also to communicate it in details to the health expert.

\section{Defining the process including the validation}

This is not a design challenge per se, but the lack of process complicates the design. Indeed, an overall process guides the design by providing sequential or parallel steps to follow, it assures that the necessary tasks are done in the right order. At our knowledge, there is no extensive process specific to the game design of therapeutic games. As a result, game designers and other involved experts have to define a process.

Here, the solution is straightforward, we need to define a design process that integrate the particularities of therapeutic game design.

The most important of these particularities is that the therapeutic efficiency of the game can not be tested regularly. Indeed, games are generally designed through short iterative phases, the game being regularly playtested to improve the design. For therapeutic games, the process has to integrate the fact that the feedback loop on the medical aspects will be long as these evaluations will be conduced at strategical moments, while the feedback loop about the game will still be short as playtests are less costly to organise.

As a result, the design must be particularly well-thought and at least theoretically validated by health experts. It means that: 1. the design must rely on precise and relevant information about the patient and the therapy, and 2. the design of the gameplay must be validated by the health expert. Thus, our process includes preliminary and intermediate steps according to these objectives.

\section{COMPONENTS OF THE METHOD}

\section{A. Model of the patient's abilities to play}

To design the gameplay, the game designer needs to know what the patients are able to do in a game. Existing models of human functioning and diagnosis questionnaires are usually very complex and not specific on abilities to play. Thus, we created a model of human functioning adapted to video game.

The model of the patient's abilities to play contains a selection of 30 abilities such as: working memory, understanding of language, fine motor control, visual acuity, and form recognition. For each of these abilities, the model gives a short definition of the ability and examples of game situations in which they are needed.

While completing the model, the health expert can rate the level of functioning of the ability by selecting one or more elements of a 7-scale (from very deficient to a lot more efficient, relative to a normal functioning adult). The objective is to get an approximation of the average level of functioning for this type of patient, but also to know the delta that may exist inter-patient. For instance, the memory can be more or less deficient depending on the Alzheimer's disease stage. The health expert can also add a description about the deficiency and rate his/her level of confidence. The model also provides guidance about which precisions may be useful for each abilities (e.g. average speed to press a button, number of items that can be memorized).

\section{B. Player in-game skills evaluation tool}

The model of the patient's abilities to play gives an overview of how the patient is functioning differently, but the data will not be as precise as "the patient needs five seconds to press a button". Plus, the data is not easily extrapolated in game environment, particularly when multiple functions are involved to complete a game objective.

Our solution here is to evaluate directly in-game what the patients can do. We propose to prototype simple game situations about every ability and combination of abilities that may be affected in the patients and then to analyse their performance in each situations. The analysis should be done on the basis of data generated by the game (i.e. a log of player's actions and events in the game). To get a more comprehensive result for the game designer, we propose to compare analysis between patients and healthy people as it will give context to the numerical data. Knowing that the patient took 10 seconds to finish the task is less informative than the patient needed twice the time compared to an average healthy person. 
This method is also useful to know the inter-patient differences and how adaptable the gameplay should be. Finally, it may help to exclude from the design gameplay actions because the patients just can not do them, but also to re-include actions that had been excluded because they were considered as probably not adapted to the patients, if the patients reveal to be able to do them in the game.

\section{Gameplay actions model}

What is needed is a way of describing the gameplay with sufficient details in order to 1 . help the game designer to define every game action the player has to do to complete the game objective, 2. facilitate the design of the gameplay regarding the patient's abilities and the intended therapeutic effects, 3 . facilitate the validation of the gameplay by the health expert.

In section II-B, we explained that the gameplay encompasses every action the player does to interact with the game and particularly to complete the game objectives. For therapeutic games, because of the patients having non-standard abilities and because of the intended therapeutic effects, the gameplay needs to be thoroughly described. A detailed description is also what will help the health expert to validate the gameplay.

To thoroughly define what should be described in a gameplay, we first introduce the notions of implicit and explicit player's actions, then we present the method.

1) Implicit and explicit player's actions: A gameplay can be thought as a loop in which the player perceives a game state on the screen, analyses the game state, decides what to do, and performs the action through a game controller [28], [29]. As a result, a gameplay loop is completed through perceptual, cognitive, and motor actions.

Motor actions are the most obvious and manifest, because the gameplay actions are explicit for us and for the game system (i.e. we have knowledge of these actions when they happen). But many gameplay actions are not explicit when they happen. These actions are implicit, we can only infer their existence. For instance, when the player has to make his/her avatar jump to reach the other side of a cliff, s/he need to evaluate the cliff's width. This action is not manifest or visible, not for us, nor for the game system. We know that the action happened because the player can not succeed without this information. Another example is when a player shoots a target, we can infer that s/he saw the target.

Implicit actions also require skills and may be used to produce therapeutic effects. Other examples of implicit game actions are: identifying a game element on screen, memorizing the game rules, detecting a sound, making a choice between two options. They all rely on perceptual and cognitive skills.

As technology improves, some player's actions may not stay implicit for ever. For instance eye trackers are able to detect when the player saw something on the screen. But, from a design perspectives, the frontier between implicit and explicit actions should be drawn according to the game system and its reaction to the player actions. If the game system can know of the action by inference only or do not react to the action, then this action should be designed as an implicit actions. For instance, if a game features an eye-tracker only to analyse the player but doesn't react to the player's gaze at all, seeing a target will still be an implicit action design-wise as the explicit action of pressing a button to shoot the target is still the only one that validate the game objective.

2) Gameplay actions description: A general description of the gameplay of a rail shooter would be: the player shoots targets. Such general description is the first step of our method. After that, the main idea is to decompose it into sub-actions until there is enough details to evaluate if each of these actions are compatible with the abilities of the patient and if the required actions for the therapeutic effects are mandatory to complete the game objective. The method can also be used backward, the required actions are defined, and then the game designer adds other relevant actions to turn the task into an actual gameplay.

The method we propose here is to describe the sub-actions of the gameplay without forgetting the implicit ones. For instance, shooting a target on screen is described as a series of sub-actions: looking at the screen, detecting a new element, reminding how targets look like, identifying that the element is a target, deciding to shoot the target, calculating the trajectory of the target, moving the mouse to be on the target, pressing a button to shoot the target.

By analysing different gameplay, we found that some part of them were generic. For instance, in most games the player has to identify a target (see fig 2) or to align the mouse cursor with the target. Those generic gameplay actions are used in very different games such as first person shooter, strategy game and puzzle game.

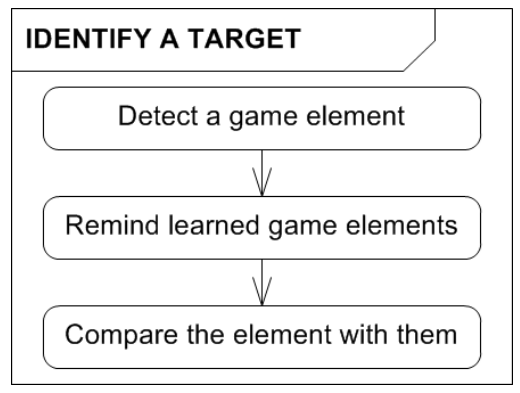

Figure 2. Generic gameplay pattern: identifying a target

With so many details on the sub-actions, it is easier to find all the parameters that can change the difficulty of a challenge. Detecting a new element on screen is easier when the element is of a different color than the background or it is harder to move the mouse on a target when the target is fast-moving.

The difficulty parameters are also what make the gameplay different when a generic gameplay actions is used in different games [30]. For instance, in puzzle games the player has more time to detect elements and align the mouse's cursor on them than in shooter games. Moreover, in shooter games, the target may be small and moving, they are part of the challenge, while in a puzzle game it is not challenging to align the cursor on game elements as it is not the purpose of such games. 
This decomposition into sub-actions facilitates the discussion with the health expert because s/he gets a clear picture of how the player interacts with the game and what aspects of the interaction can be problematic.

When problems are detected, the gameplay can be changed accordingly. For instance, if the patient is not able to press a button, it is possible to define that the game automatically shoots when the cursor is aligned with a target. Sometimes, a game action may not be taken out of the gameplay. For instance, if the patient's memory is deficient and therapy-wise it is necessary that there is different targets and non-targets (i.e. elements the player should not shoot) in the game, a solution may be to have an on-screen reminder of what targets look like. Another solution is to have less elements to memorize. In this case, the analysis of the difficulty parameters is used to adapt the game action to the player (i.e. making it easier).

\section{Game Design Method for Therapeutic Games}

Most existing game design methods are an adaptation of a more general process called design thinking. As explained by Zimmerman et al., design thinking is the three-step process designers apply [31]:

- Investigation: gaining multiple perspectives on a problem

- Ideation: generation of many possible different solutions

- Iteration: cyclical process of refining a solution

Our method conforms to this general process as reflected by its three main phases:

- Phase 1. Investigating the problem with health experts

- Phase 2. Designing the gameplay

- Phase 3. Prototyping the therapeutic game

Our method focus on the beginning of the project when most of the game design is done (i.e. the concept and preproduction phases).

\section{A. Phase 1: Investigating the problem with health experts}

During this phase, the objective is to get the health knowledge necessary to design the gameplay. We propose the use of models: the P/G/T model (section V) and the specialized sub-model of the patient's abilities to play (section VII-A).

Aside from these models, the game designers can also rely on other methods such as personas, focus groups and interview to better define the patients, their preferences and needs. Provided there exist games accessible to the patients, the game designer may even organize playtests on these games to observe directly the level of performance of the patients, but also what they like while playing.

When the game designer has all the needed information, s/he may proceed to the next phase.

\section{B. Phase 2: Designing the gameplay}

If the phase 1 was about investigating the problem and getting theoretical data, the second phase is about getting more specific data, generating solutions and validating a gameplay with health experts.

This phase is composed of three iterative steps:

- Step 2.1. Gameplay design and formalisation
- Step 2.2. Gameplay prototyping

- Step 2.3. Playtest

In step 2.1., the game designer can use the method we propose in section VII-C to design and formalize a gameplay. S/he may then validate it with health experts.

Then, in step 2.2., the gameplay should be quickly prototyped. Then, the prototype is tested with patients in step 2.3. We proposed guidelines regarding this kind of prototype in section VII-B.

It is not mandatory to start with the first step. Depending on the project, it can be more relevant to start with step 2.2. to collect more information about the patient first. This is done by prototyping generic gameplay actions and test them first on patient to gain better knowledge of what they are able to do in games before starting the actual gameplay design.

When the gameplay is evaluated as being probably therapeutic by an health expert and the prototype achieves its objective in terms of accessibility and enjoyability, the phase 3 may begin.

\section{Phase 3: Prototyping the therapeutic game}

This phase is similar to a video game preproduction, the objective is to produce and evaluate a pre-production prototype before starting the production. The prototype should be of final quality to evaluate its motivating effects on the patient, but also contains enough contents to allow for medical evaluation of its therapeutic effects.

This phase is composed of three iterative steps:

- Step 3.1. Game Design

- Step 3.2. Game prototyping

- Step 3.3. Medical validation \& playtests

In phase 3, the gameplay should already be solid as it has been playtested for its accessibility and fun, and at least theoretically validated for its therapeutic effects in phase 2 .

During step 3.1., the objective is to design the other aspects of the game and to address as best as possible the patient's motivation by including features such as a compelling game world and story. For this phase, we will propose a list of motivating features in games categorized in challenge, game world, and social interactions. This list will be the subject of a latter publication.

The prototype is developed in step 3.2. and intermediate playtests may take place to improve the design before the more thorough validation of the step 3.3.

The step 3.3. concerns the medical evaluation of the therapeutic effects, along with playtests to evaluate if the game is motivating for the patients. When the prototype achieves the intended results, the production may begin.

\section{DISCUSSION AND FUTURE WORKS}

We identified four therapeutic game design challenges and addressed them through at least partial solutions taking the form of a method providing a process, a method to formalize the gameplay, and two different models (P/G/T model and player's abilities to play model). 
However, this design method for therapeutic game along with its components can still be improved. Particularly, the gameplay action model still need work to become at least semi-formal to facilitate the exchange of knowledge between game designers.

Also, our work did not explore participatory design with health experts and with patients as possible solutions. In our methods, patients are studied, but not involved, and health experts have the role of providing knowledge and validating the design. So one of our next step will be to propose at least optional steps of participatory design.

Moreover, we conduced some tests with students to observe how they reacted to the method and how it affected their design. If we observed that they were more attentive to the patient's abilities and the general accessibility of the game, it seems that the way we convey the method is not efficient enough. For instance, we are not sure if the students understood our method to design the gameplay. Thus, comprehensive materials to explain how to use the method should now be produced.

Another way to convey our method would be to embed it into a design software. Such design software would also be an opportunity to compute and centralize the data of a therapeutic game project. For instance, the patient's abilities to play model could be automatically updated with data from the prototype testing their abilities.

We may also aggregate data from different projects to propose insights and advices. For instance, when the game designer enters the age of the patient in the model, the software may highlight abilities that are generally affected in this age group by comparing data of similar projects.

As a consequence, designing and developing a software that embed our method and tools is the next big step of our research. We believe that such software may help game designer to better design therapeutic games, but also may help us to discover new knowledges on therapeutic games and on how to design them. In the long-term, we may even be able to directly link specific game actions or combination of game actions to therapeutic effects, as well as getting precise models of player's abilities to play for certain disabilities and diseases. The use of these knowledge may then greatly improve and accelerate the design phase of therapeutic games by making the gameplay design more evidence-based.

\section{REFERENCES}

[1] J. McGonigal, Reality is broken. New York: Penguin Books,, 2011.

[2] E. Sabaté and W. H. Organization, Adherence to Long-term Therapies: Evidence for Action, ser. Academic Search Complete. World Health Organization, 2003.

[3] S. F. Bassett, "The assessment of patient adherence to physiotherapy rehabilitation," New Zealand journal of physiotherapy, vol. 31, no. 2, pp. 60-66, 2003.

[4] P. M. Kato, "Video games in health care: Closing the gap." Review of General Psychology, vol. 14, no. 2, p. 113, 2010.

[5] J. W. Burke, M. D. J. McNeill, D. K. Charles, P. J. Morrow, J. H. Crosbie, and S. M. McDonough, "Optimising engagement for stroke rehabilitation using serious games," Vis. Comput., vol. 25, pp. 10851099, October 2009.
[6] J. A. Anguera, J. Boccanfuso, J. L. Rintoul, O. Al-Hashimi, F. Faraji, J. Janowich, E. Kong, Y. Larraburo, C. Rolle, E. Johnston et al., "Video game training enhances cognitive control in older adults," Nature, vol. 501, no. 7465, pp. 97-101, 2013.

[7] H. G. Hoffman, J. N. Doctor, D. R. Patterson, G. J. Carrougher, and T. A. Furness, "Virtual reality as an adjunctive pain control during burn wound care in adolescent patients," Pain, no. 85, pp. 305-309, 2000.

[8] E. Adams, Fundamentals of Game Design, second edition. New Riders Publishing, 2009.

[9] T. McGraw, K. Burdette, and K. Chadwick, "The effects of a consumeroriented multimedia game on the reading disorders of children with adhd. 2005-6," in Proceedings of DiGRA 2005 Conference: Changing Views-Worlds in Play, Vancouvor, Canada, 2005.

[10] R. Hunicke, M. Leblanc, and R. Zubek, "Mda: A formal approach to game design and game research," in In Proceedings of the Challenges in Games AI Workshop, Nineteenth National Conference of Artificial Intelligence. Press, 2004, pp. 1-5.

[11] K. Salen and E. Zimmerman, Rules of Play : Game Design Fundamentals. The MIT Press, October 2003.

[12] T. Fullerton, Game Design Workshop: A Playcentric Approach to Creating Innovative Games. CRC Press, 2008.

[13] A. Rollings and E. Adams, Andrew Rollings and Ernest Adams on game design. New Riders, 2003.

[14] B. Brathwaite and I. Schreiber, Challenges for game designers. Course Technology, 2009.

[15] J. Schell, The Art of Game Design: A book of lenses. CRC Press, 2008.

[16] S. Rogers, Level Up!: The Guide to Great Video Game Design. John Wiley \& Sons, 2010.

[17] R. Bartle, "Hearts, clubs, diamonds, spades: Players who suit muds," Journal of MUD Research, 1996.

[18] N. Yee, "Motivations for play in online games," CyberPsychology \& behavior, vol. 9, no. 6, pp. 772-775, 2006.

[19] T. W. Malone, "What makes things fun to learn? heuristics for designing instructional computer games," in SIGSMALL '80: Proceedings of the 3rd ACM SIGSMALL symposium and the first SIGPC symposium on Small systems. New York, NY, USA: ACM, 1980, pp. 162-169.

[20] S. Benveniste, "Incremental design of therapeutic music games: Theory and application to the treatment of behavioral disorders and alzheimer's disease," Ph.D. dissertation, MINES ParisTech, 2010.

[21] V. V. Abeele, B. De Schutter, L. Geurts, S. Desmet, J. Wauters, J. Husson, L. Van den Audenaeren, F. Van Broeckhoven, J.-H. Annema, and D. Geerts, "P-iii: A player-centered, iterative, interdisciplinary and integrated framework for serious game design and development," in Serious Games: The Challenge. Springer, 2012, pp. 82-86.

[22] B. Marne, B. Huynh Kim Bang, and J.-M. Labat, "Articuler motivation et apprentissage grâce aux facettes du jeu sérieux," in Environnements Informatiques pour l'Apprentissage Humain, 5 2011, pp. 69-80, isbn: 978-2-87325-061-4.

[23] S. Mader, "Le game design de jeux thérapeutiques : Modèles et méthodes pour le design du gameplay," Ph.D. dissertation, CEDRIC Laboratory, Paris, France, 2015.

[24] S. Mader, S. Natkin, and G. Levieux, "How to analyse therapeutic games: The player / game / therapy model," in Entertainment Computing ICEC 2012, ser. Lecture Notes In Computer Sciences, Springer, Ed., 2012.

[25] D. Ghozland, "Designing for motivation," Gamasutra, 2007.

[26] S. Rigby and R. Ryan, "Rethinking carrots: A new method for measuring what players find most rewarding and motivating about your game," Gamasutra. com, 2007.

[27] S. Mader, J. Dupire, S. Natkin, and E. Guardiola, "Designing therapeutic games for seniors: case study of "le village aux oiseaux" (birds village)," Modelling, Measurement and Control, vol. 73, no. 3, p. n.c., 2012.

[28] T. Heaton, "A circular model of gameplay," Gamasutra. com, 2006.

[29] B. Perron, "The heuristic circle of gameplay: the case of survival horror," Gaming realities: A challenge for digital culture, pp. 62-71, 2006.

[30] G. Levieux, "Mesure de la difficulté dans les jeux vidéo." Ph.D. dissertation, Conservatoire National des Arts et Métiers, 2011.

[31] J. Zimmerman, J. Forlizzi, and S. Evenson, "Research through design as a method for interaction design research in hci," in Proceedings of the SIGCHI conference on Human factors in computing systems. ACM, 2007, pp. 493-502. 